\title{
Road Network Modelling for Maintenance Planning
}

\author{
C. Yang, R. Remenyte-Prescott \& J. Andrews \\ University of Nottingham, United Kingdom
}

\begin{abstract}
In this paper a novel road network flow model is presented, suitable for modelling both motorway and urban roads. A road network is modelled using a directed graph, consisting of typical roads and junctions, with different traffic flow inputs throughout the day. The model is based on a simple idea of balancing out the traffic flow in the network, considering the capacity of an individual road and traffic overflow through to the related junctions. The evolution of traffic flows and queues in a two-way traffic flow is modelled using an iterative simulation method. The model is used to forecast travel delays and evaluate the network performance in normal conditions and when maintenance works are implemented. The paper illustrates how to calculate travel delays on the network throughout the day, how to estimate the effects of road maintenance works and how to use the results to plan maintenance on the network.
\end{abstract}

\section{INTRODUCTION}

In recent years highways agencies have been focusing on maintaining and rehabilitating existing roads instead of constructing new ones. Billions of pounds are spent yearly to maintain UK roads at the required standard (A.I.A. 2011). Travel delay cost due to maintenance is significant and can substantially increase the corresponding maintenance cost. Hence it is of great importance to consider road user cost when planning maintenance. Due to roadworks traffic flow rates are restricted and queues on the network can occur. Therefore, a road flow model can be used to describe travel delays on the network and to compare the effects of different maintenance policies.

Typically, two main groups of models for road networks are developed - microscopic and macroscopic. Microscopic models consider the travel behaviour of individual vehicles, whereas macroscopic models are used to describe the aggregate behaviour of vehicles. Driver behaviour in traffic is difficult to observe and measure and computational effort required for a microscopic model can be high. A macroscopic model, proposed in this paper, is more suitable for network-level modelling due to good realtime quality, the ability to consider the network as a whole and generally good availability of data.

A number of different macroscopic models have been developed to model traffic on motorways and urban roads. These include a pioneering flowdynamic model by Lighthill \& Whitham (1955), its approximation by Daganzo (1994), METANET model by Messmer \& Papageorgiou (1990), and further extensive research when introducing variable speed limits by Breton et al. (2002) and Hegyi et al. (2005) and when introducing route guidance by Deflorio (2003) and Karimi et al. (2004) on a motorway network. In 2007 Van den Berg et al. developed a model for mixed urban and motorway networks, since motorway traffic is heavily influenced by the traffic flows on the connected urban roads, and vice versa.

The model in this paper considers additional properties of a road network structure, such as priority junctions, which were not considered in the previous work. Shared lanes where traffic heading to different directions is mixed together are used to obtain a more realistic network representation, instead of assigning subqueues for each direction of turning. Both motorway and urban junctions are modelled following the same principle, i.e. a maximum flow rate at the junctions where flows compete is considered in order to derive delay times on the network. Finally, in addition to the previous work on one-way traffic flow models, two-way traffic flow along network links is modelled in this approach. Finally, roadwork nodes are introduced for maintenance modeling.

The main steps of how to build the model and evaluate the network are given in Section 2, together with an overview of the models for the main junction types. Data requirements are discussed in Section 3 and the case study of a medium-sized network 
in the UK is presented in Section 4 in order to show the applicability of the model to calculate traffic delays on a real network. Section 5 contains an overview of the model application for maintenance planning, illustrated by some results, and conclusions and future work are discussed in Section 6.

\section{ROAD NETWORK FLOW MODEL}

\subsection{Network representation}

A network model, proposed in this paper, consists of nodes, which represent junctions, and links, which represent roads. Junction models contain links which model the exit traffic from one junction to enter the next junction and vice versa, therefore, two-way traffic is considered. There can be a number of source and sink nodes on the network, which represent the flow into network and out of network respectively, also known as the edges of the network. In addition, the roads themselves can have source and sink nodes, which model traffic entering/leaving the road. This represent significant traffic from/to such elements as housing estates, places of work, airports and railway stations. This feature of the model assists in avoiding the inclusion of all minor roads on the network. A case study network is shown in Figure 2 in Section 4.

\subsection{Algorithm}

The idea of the algorithm is to calculate average queues per time step on the network, when the number of cars per time step exceeds the capacity of the link and queues overflow to the related junction. The evolution of two-way traffic is performed using an iterative simulation method to calculate traffic flows and queues on the network.

First of all, the flow on each link is calculated by passing flows from the source nodes to the sink nodes. If the flow exceeds the flow capacity of the link, i.e. the rate of incoming vehicles is higher than the rate of outgoing vehicles, the queue is calculated. If the queue exceeds the capacitance of the link, i.e. the queue of cars is longer than the link, the queue is propagated back through the network. This process is repeated until the convergence is reached, i.e. the flows on each link do not change by further iterations. For every time step the same process is repeated and the aggregate queues on the network are calculated. Throughout the day, the queues can increase and even "overspill" to other incoming links, for example, in the rush hour, or the queues can decrease and the network eventually become clear of queues, for example, due to a longer duration of green phase on traffic lights. Following this principle traffic performance on a given network can be modelled. A detailed set of rules are presented below.

\subsection{Mathematical model}

Flow on the link $i, j$ at time $t_{k}$ is calculated as a sum of all the flows to node $i$, the positive source flow entering the link and the negative sink flow leaving the link:

$$
f_{i, j}\left(t_{k}\right)=\sum_{\text {alls }} d_{s, i, j}\left(t_{k}\right) f_{s, i}\left(t_{k}\right)+s r_{i, j}\left(t_{k}\right)-s k_{i, j}\left(t_{k}\right)
$$

where $f_{i, j}\left(t_{k}\right)=$ flow on the link at time $t_{k}$, (passenger car unit (pcu)/hour), $d_{s, i, j}\left(t_{k}\right)=$ proportion of flow on the link coming from the direction $s$ at time $t_{k}$, $s r_{i, j}\left(t_{k}\right)=$ source flow entering the link at time $t_{k}$, (pcu/hour), and $s k_{i, j}\left(t_{k}\right)=$ sink flow leaving the link at time $t_{k}$, (pcu/hour).

Once the flow on each link at time $t_{k}$ is calculated, the flow and the queue values are updated according to the flow capacity, $c_{i, j}$, and the link capacitance, $c p_{i, j}$, if necessary. If the flow on the link is higher than the flow capacity, i.e. $f_{i, j}\left(t_{k}\right)>c_{i, j}$, the surplus in cars is placed in a queue, applying Equations 2 and 3:

$$
\begin{aligned}
& f_{i, j}\left(t_{k+1}\right)=c_{i, j} \\
& q_{i, j}\left(t_{k+1}\right)=q_{i, j}\left(t_{k}\right)+\left(f_{i, j}\left(t_{k}\right)-c_{i, j}\right) \cdot \Delta t
\end{aligned}
$$

where $\Delta t=$ length of time step, (hour), and $q_{i, j}\left(t_{k}\right)=$ average number of vehicles queuing on the link at time $t_{k}$, (pcu). Also note that $q_{i, j}\left(t_{k+1}\right)$ is used for the updated value of $q_{i, j}\left(t_{k}\right)$. An additional notation for the updated value is avoided, following the assumption that if the queue is present at time $t_{k}$, it is also present at the beginning of the next time step $t_{k+1}$.

In addition, if the queue is higher than the capacitance, i.e. $q_{i, j}\left(t_{k}\right)>c p_{i, j}$, the surplus in cars is recorded to be passed back to the links where the cars came from, applying Equations 4 and 5:

$q_{i, j}\left(t_{k}\right)=c p_{i, j}$

$q_{i}\left(t_{k}\right)=q_{i, j}\left(t_{k}\right)+\left(f_{i, j}\left(t_{k}\right)-c_{i, j}\right) \cdot \Delta t-c p_{i, j}$

where $q_{i}\left(t_{k}\right)=$ queue at the end of the link at time $t_{k}$.

If the flow is lower than the flow capacity and there is a queue at time $t_{k}$, i.e. $f_{i, j}\left(t_{k}\right)<c_{i, j}$ and $q_{i, j}\left(t_{k}\right)>0$, the queue decreases, applying Equations 2 and 3, and can completely disappear. In the latter case, Equations 6 and 7 are used:

$$
\begin{aligned}
& f_{i, j}\left(t_{k}\right)=c_{i, j}+\frac{q_{i, j}\left(t_{k}\right)}{\Delta t} \\
& q_{i, j}\left(t_{k+1}\right)=0
\end{aligned}
$$


The process of the queue propagation is simply described as passing back the proportion of the queue to each link, which equals the proportion of the flow that contributed to the overall flow in the first place. For example, if a queue builds up on the link between nodes $j$ and $j+1$ and it exceeds the capacitance of the link by the number of vehicles, $q_{j}\left(t_{k}\right)$, it is proportionally distributed back to all the links that enter node $j$. This process increases the size of the queue and decreases the flow on each link that enters node $j$, as shown below:

$q_{i, j}\left(t_{k+1}\right)=q_{i, j}\left(t_{k}\right)+\frac{f_{i, j}\left(t_{k}\right)}{f_{j, j+1}\left(t_{k}\right)} \cdot q_{j}\left(t_{k}\right)$

$f_{i, j}^{\prime}\left(t_{k}\right)=f_{i, j}\left(t_{k}\right)-\frac{f_{i, j}\left(t_{k}\right)}{f_{j, j+1}\left(t_{k}\right)} \cdot \frac{q_{j}\left(t_{k}\right)}{\Delta t}$

where $f_{i, j}^{\prime}\left(t_{k}\right)=$ updated flow on the link at time $t_{k}$, (pcu/hour).

The process of queue propagation is carried out through the network until the queue can be accommodated and it does not exceed the capacitance of the link. Note that nodes on the edges of the network are assigned to infinite flow capacity, so that the process of queue propagation is terminated at the boundaries of the network.

\subsection{Junction models}

A range of junction models, as shown in Table 1, are constructed in order to describe different traffic interactions on the network.

Table 1. Junction types.

\begin{tabular}{ll}
\hline Junction groups & Junction types \\
\hline Signalised & $\begin{array}{l}\text { Signalised T-junction, signalized } \\
\text { intersection, signalized roundabout }\end{array}$ \\
\hline Priority & $\begin{array}{l}\text { T-junction, urban roundabout, motorway } \\
\text { roundabout }\end{array}$ \\
\hline One-way & $\begin{array}{l}\text { On-ramp and off-ramp, merge and } \\
\text { diverge, roadwork node }\end{array}$ \\
\hline
\end{tabular}

Some less commonly modelled junctions are explained below. For example, on a motorway roundabout cars can queue on the roundabout itself, when the exit from the roundabout to the motorway is blocked and cars are queuing to join the motorway. On-ramp and off-ramp junctions are used to model slip roads for entering and exiting the motorway. Finally, a roadwork node represents a part of the road under maintenance. These junctions are introduced in order to be able to model a road network as close to reality as possible.
A common feature of the junction models is that conflicting flows affect the flow throughout the network and they need to be considered while developing the models. For example, traffic flow at a signalised junction is influenced not only by the flow capacity of the entry links, but also by the green split duration of the signals. For priority junctions the traffic follows the right-of-way rules, when the entering flow is restricted not only by the flow capacity of the link but also by the traffic flows from the competing links. For example, T-junction model is developed using the example in Figure 1.

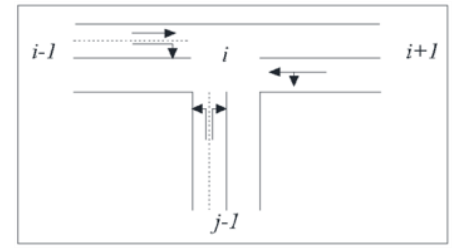

Figure 1. T-junction model.

While developing the model, the three roads in Figure 1 are considered one by one. For the major road $i-1$ the flow to $i+1$ is not restricted by any conflicting traffic, whereas the flow to $j-1$ is restricted by the flow from $i+1$ to $i$. For the flow from direction $i+1$ no restrictions from the conflicting traffic exist. For the flow from $j-1$ a number of different conflicting flows are considered. If conflicting traffic exists, it is considered while calculating the length of the queue, otherwise, only restrictions upstream the network influence the queue.

Roadwork nodes are introduced on the network in order to be able to investigate maintenance effects on traffic delays. Since three common types of roads are modelled, i.e. a single carriageway, a dual carriage way and a motorway, three types of roadwork nodes are used to represent possible maintenance arrangements. For example, on a single carriageway traffic flows from the two directions have to share the single lane in service, commonly controlled by traffic lights when maintenance is carried out. Since the traffic can pass the worksite only through the green splits in the relevant direction, the model takes account of not only the length of the closed road but also of the duration of green splits. Similarly, on a dual carriageway or a motorway one or two lanes can be closed for maintenance and speed restrictions are commonly introduced on the lane in service. Therefore, the reduced flow capacity passing the worksite, as well as the length and the width of the closed road, can be modelled. This flexibility of the model is of great importance, when different maintenance arrangements are analysed.

\subsection{Network performance metrics}

Network performance is evaluated taking account of the results obtained on the individual junctions and 
links. It is expressed in terms of the total travel duration spent on the network at each time step $t_{k}$ :

$$
\begin{aligned}
& \operatorname{TTD}\left(t_{k}\right)=\sum_{i=1}^{N} T_{D, i}\left(t_{k}\right)+\sum_{j=1}^{M} T_{T, j}(t)_{k} \\
& T_{D, i}\left(t_{k}\right)=\sum_{a=1}^{A_{i}} \int_{t_{k-1}}^{t_{k}} q_{a, i}(t) d t
\end{aligned}
$$

where $\operatorname{TTD}\left(t_{k}\right)=$ total travel duration on the network at time $t_{k}$, (hour), $T_{D, i}\left(t_{k}\right)=$ total delay time at junction $i$ at time $t_{k}$, (hour), $T_{T, j}\left(t_{k}\right)=$ total travel time on link $j$ at time $t_{k}$, (hour), $q_{a, i}\left(t_{k}\right)=$ average number of vehicles queuing on arm $a$ of junction $i$ at time $t_{k}$, (pcu), $N=$ number of junctions on the network, $M=$ number of links on the network and $A_{i}=$ number of arms at junction $i$.

Note, that the arms of the junction are the links entering and exiting the junction. Also, the total travel time on link $j, T_{T, j}\left(t_{k}\right)$, is calculated considering the travel time for the traffic that passes the link without delays and the time spent queuing.

Finally, the total road user cost for the network at time $t_{k}$ can be evaluated:

$$
C_{u}\left(t_{k}\right)=T T D\left(t_{k}\right) \times v
$$

where $C_{u}\left(t_{k}\right)=$ total road user cost at time $t_{k}, v=$ time value for road user per hour. According to 2012 prices the market price value of time for an average vehicle is $£ 15.38$ per hour (DfT 2012).

\section{DATA SOURCES}

In order to calculate traffic delays using the proposed method, a number of different data inputs are needed, including the geographical characteristics of the road network along with the traffic flows through it at different times of the day. In terms of geographical network features, the length for each link and the flow capacity for each arm of the junction are required. In addition, the traffic volume entering each link of the network throughout the day are required, along with the proportion of vehicles leaving each junction on each of the exit arms and the signal control inputs at each time interval. Note that in this model traffic flows and turning movements are constant over each time step. For maintenance modelling the locations and the arrangements of maintenance works are needed, including the type of the road under maintenance, the length of the worksite, the duration of works, the speed restrictions, etc.

The available traffic data inputs on the highway network, discussed in the following section, were the number of cars per hour, which had been collected at various locations on trunk roads and at various junc- tions over the last couple of years. Such traffic data were obtained from the Highways Agency and Nottingham County Council (Highways Agency 2011) for the majority of the roads, and were directly applied to model two-way traffic flow during a day. Also, some data collection was carried out by the authors if necessary data were not available.

\section{CASE STUDY}

In order to illustrate the performance of the road network flow model on a real highway network and its suitability to predict travel delays and estimate different road maintenance approaches, a case study based on a medium-sized network in the UK is presented. The chosen network between Loughborough and Nottingham includes both urban roads and motorways.

Only trunk roads and roads between major junctions are retained in the network, as shown in Figure 2. Some symbols, e.g. M1 and A52, denote the road class and road number of the road links, where $M$ represents a motorway and A - a trunk road. Other symbols represent the type and ID of a junction, for instance, a "diverge" junction in Loughborough is named D2. There are 47 junctions modelled in the network, including 8 roundabouts (R), 3 signalised roundabouts (SR), 5 diverge junctions (D), 3 merge junctions (MG), 12 off-ramps and 12 on-ramps (both denoted by S), 2 signalised T-junctions (ST), and 2 signalised intersections (SI); and 51 road links, including 4 motorway links, 1 single carriageway link (SR1-ST1) and 46 dual carriageway links.

\subsection{Example network performance}

The first objective of this case study is to test the method on the example network, predict the outflows throughout the day and identify weak areas of the network which experience severe traffic congestion. For the purpose of the illustration, the algorithm was executed for 16 one-hour steps on a working day between $7 \mathrm{am}$ to $11 \mathrm{pm}$ and it took less than 10 seconds to obtain the results. As it is seen in Table 2, the analysis showed that the roundabouts $\mathrm{R} 1$, R6 and R7 and their incoming links were badly congested during the morning and evening peak times, whereas the flow throughout the rest of the network was not disturbed. The three roundabouts are located on the city ring road, and are usually badly congested at these times, as also confirmed by the model. 


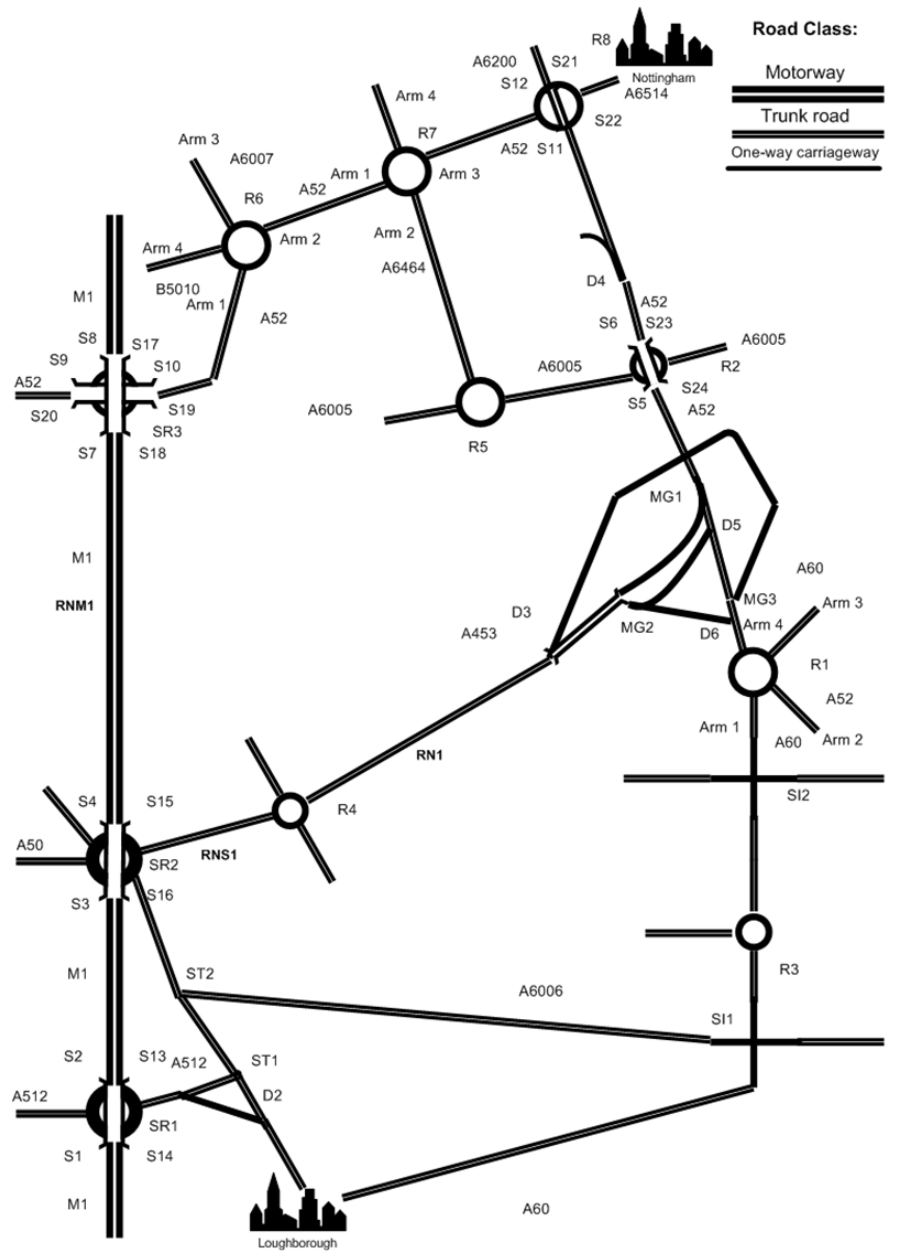

Figure 2. Case study network.

Table 2. Traffic condition on the example network.

\begin{tabular}{|c|c|c|c|c|c|}
\hline \multirow[t]{2}{*}{ Time } & \multicolumn{5}{|c|}{ Traffic condition } \\
\hline & $\operatorname{Arm1}(\mathrm{R} 1)$ & SI2-R1 & Arm3(R6) & Arm4(R6) & Arm4 (R7) \\
\hline 7 & NQ & NQ & NQ & NQ & NQ \\
\hline 8 & MINQ & NQ & NQ & MAJUQ & MÄJQ \\
\hline 9 & MAJQ & MINC & NQ & MAJQ & NQ \\
\hline 10 & MAJQ & MAJC & MÄJQ & NQ & $\mathrm{NQ}$ \\
\hline $11-16$ & NQ & NQ & NQ & NQ & NQ \\
\hline 17 & NQ & NQ & NQ & MAJQ & MAJQQ \\
\hline 18 & NQ & NQ & NQ & MAJQ & MAJQ \\
\hline 19 & NQ & NQ & MAJQ & NQ & NQ \\
\hline $20-22$ & NQ & NQ & NQ & NQ & $\mathrm{NQ}$ \\
\hline
\end{tabular}

Note that NQ stands for no queue formed in the link, MINQ - minor queue (under $100 \mathrm{pcu}$ ), MAJQ - major queue (more than $100 \mathrm{pcu}$ ).

When all the queues on the network are added at each time step to represent the overall network performance, it can be seen that the queues build up at morning and evening peak times, as shown in Figure 3.
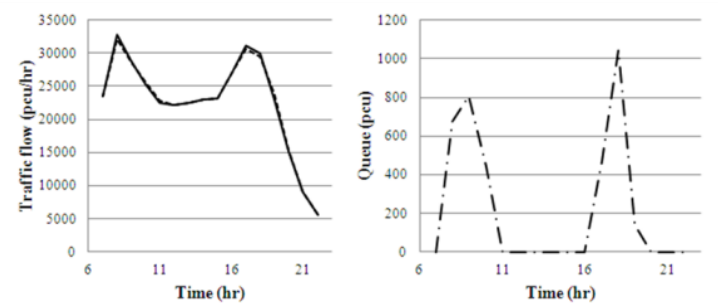

- Inflow(pcu hr) -----Outflow (peu hr $-\cdots$ Queue (pcu)

Figure 3. Traffic condition when no maintenance is performed
On the left hand side of Figure 3 the total flow into the network and out of the network has been presented, with the expected increase at peak times. On the right hand side the sum of all the queues formed in the network throughout the day is given.

After the method has been applied to identify the congested areas of the network, further research has been carried out to develop rules of traffic rerouting in the network in order to minimise traffic delays. This research has been developed by the authors but is not the scope of the paper.

\subsection{Modelling maintenance effects on the network}

The second objective of this case study is to model maintenance effects on the network and to calculate additional travel delays caused by roadworks. In order to model maintenance works on the network, roadwork nodes are introduced in the network, as discussed in Section 2.4. For the purpose of the illustration, consider a single carriageway in the example network, SR2-R4, and its worksite RNS1, as shown in Figure 2. The results of the analysis are given in Figure 4.
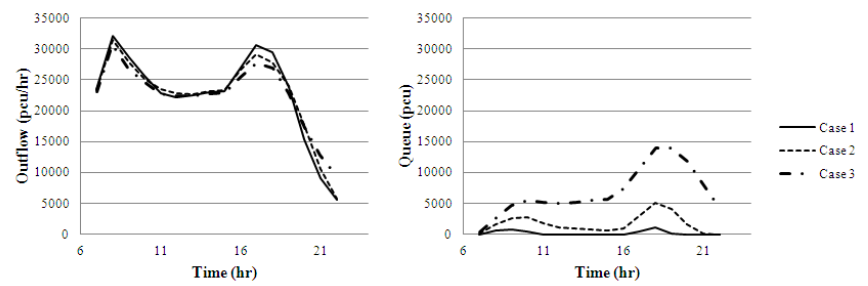

Figure 4. Traffic condition when maintenance is performed

Three cases are considered: in case 1 no maintenance is performed, in case 2 the green splits for both directions of traffic passing the worksite are $45 \%$ and in case 3 the green splits are $40 \%$, since the worksite is assumed to be twice as long as in case 2 . The three cases were chosen to illustrate the capability to model different maintenance arrangements. If required, more than one instance of maintenance can be modelled on the network at the same time. As expected, queues on the network during maintenance are longer than without maintenance, especially, when in the example network only one side of the single carriageway is used for the two-way traffic, since the other side is closed for maintenance. In addition, shorter green splits in case 3 also cause longer queues.

The results confirm the fact that roadworks have a great impact on network performance, especially, when the traffic flow along a road is greater than its capacity, usually observed at peak times. By analysing the delays caused by maintenance, roadworks can be planned to cause less disruption when possible, for example, carry out road maintenance when the traffic is low. However, additional factors, such as the cost of maintenance at night or the set-up cost if maintenance is done in intervals during the course 
of a few days, could be considered in maintenance planning.

\section{MODEL APPLICATION FOR MAINTENANCE PLANNING}

The proposed model could be used as a tool to evaluate different maintenance actions and their effects on the network. For example, different start times of maintenance during the day and different maintenance worksite arrangements, such as the number of closed lanes and traffic controls of flows, can be evaluated. Such results can then be used by an asset management team while planning road network maintenance. In a simple case, the total cost of works can be estimated, which consists of highway agency cost and road user cost, and the option with the lowest cost can be proposed. Following such an approach the best way of minimising the traffic congestion when necessary maintenance is carried out can be selected. The model can also be applied to support more complex maintenance decisions, as discussed at the end of this section.

\subsection{Examples of minimising cost}

A small example network, containing 6 junctions and 10 links, is used to demonstrate the concept of how the model can be used to plan road maintenance. The daily network performance when no maintenance is carried out is shown in Table 3 . The traffic data and maintenance cost are assumed in this example and they are illustrative of the quantities given in literature.

Table 3. Network performance without maintenance.

\begin{tabular}{lllclccl}
\hline Time Inflow & Outflow & Queue & \multicolumn{3}{l}{$\begin{array}{l}\text { Journey Travel } \\
\text { time }\end{array}$} & TTD & $C_{u}$ \\
& & & & & & & \\
\hline $7-8$ & 10000 & 10000 & 0 & 538 & 0 & 538 & 8267 \\
$9-15$ & 5000 & 5000 & 0 & 269 & 0 & 269 & 4133 \\
$16-18$ & 10000 & 10000 & 0 & 538 & 0 & 538 & 8267 \\
$19-21$ & 5000 & 5000 & 0 & 269 & 0 & 269 & 4133 \\
22 & 2000 & 2000 & 0 & 108 & 0 & 108 & 1653 \\
23 & 1000 & 1000 & 0 & 54 & 0 & 54 & 827 \\
$0-5$ & 100 & 100 & 0 & 5 & 0 & 5 & 83 \\
6 & 1000 & 1000 & 0 & 54 & 0 & 54 & 827 \\
\hline
\end{tabular}

Since no delays occur throughout the day, the example network is sufficient for carrying the traffic on the network.

Assume that a single carriageway needs to be closed for 10 hours of maintenance, and the works cost $£ 6250$. The model is applied to find the best start time of works during the day. For example, if the works were to start at $9 \mathrm{am}$, i.e. at the end of the morning peak time, some queues will occur, as illus- trated by the results of network performance in Table 4.

Table 4. Network performance with maintenance (start time 9am).

\begin{tabular}{|c|c|c|c|c|c|c|c|}
\hline \multicolumn{2}{|c|}{ Time Inflow } & Outflow & \multirow{2}{*}{$\begin{array}{c}\text { Queue } \\
0\end{array}$} & \multirow{2}{*}{$\begin{array}{l}\begin{array}{l}\text { Journey } \\
\text { time }\end{array} \\
538\end{array}$} & $\begin{array}{l}\text { Travel } \\
\text { delay }\end{array}$ & \multirow{2}{*}{$\begin{array}{r}\text { TTD } \\
538\end{array}$} & \multirow{2}{*}{$\begin{array}{r}C_{u} \\
8267\end{array}$} \\
\hline $7-8$ & 10000 & 10000 & & & 0 & & \\
\hline $9-15$ & 5000 & 5000 & 0 & 272 & 0 & 272 & 4191 \\
\hline 16 & 10000 & 9624 & 376 & 520 & 189 & 708 & 10890 \\
\hline 17 & 10000 & 9412 & 964 & 519 & 672 & 1191 & 18322 \\
\hline 18 & 10000 & 9355 & 1609 & 527 & 1291 & 1817 & 27946 \\
\hline 19 & 5000 & 6609 & 0 & 384 & 297 & 681 & 10476 \\
\hline $20-21$ & 5000 & 5000 & 0 & 269 & 0 & 269 & 4133 \\
\hline 22 & 2000 & 2000 & 0 & 108 & 0 & 108 & 1653 \\
\hline 23 & 1000 & 1000 & 0 & 54 & 0 & 54 & 827 \\
\hline $0-5$ & 100 & 100 & 0 & 5 & 0 & 5 & 83 \\
\hline 6 & 1000 & 1000 & 0 & 54 & 0 & 54 & 827 \\
\hline
\end{tabular}

When maintenance is carried out, the road user cost increases significantly and some queues occur on the network. As discussed in the case study in section 4 , this is due to the fact that the two-way traffic uses the single available lane due to closures, and the traffic flow on some links exceeds their capacitance, especially observed at evening peak times. The total cost of the proposed maintenance arrangement, i.e. start the closure of the single carriageway at 9am, is calculated by adding the road user cost and the maintenance cost, which is $£ 125572$. However, the delays can be minimised by choosing a different start time of works and minimising the total cost, as illustrated in Figure 5.

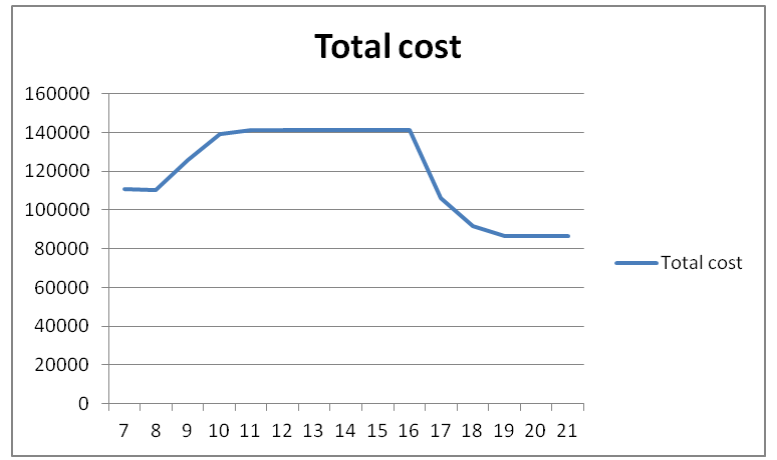

Figure 5. Total cost for different start time.

The maintenance start at $9 \mathrm{pm}$ results in the minimum total cost, since the traffic flow overnight is low. Note that a potential increase of the maintenance cost overnight is not considered in this example.

In addition, the model can be used to choose other worksite arrangements in order to minimise traffic delays. For example, Figure 6 illustrates that the green splits during the maintenance of a single carriageway can be optimised. In this example, the best green splits are in the interval $[40 \%, 50 \%]$. 


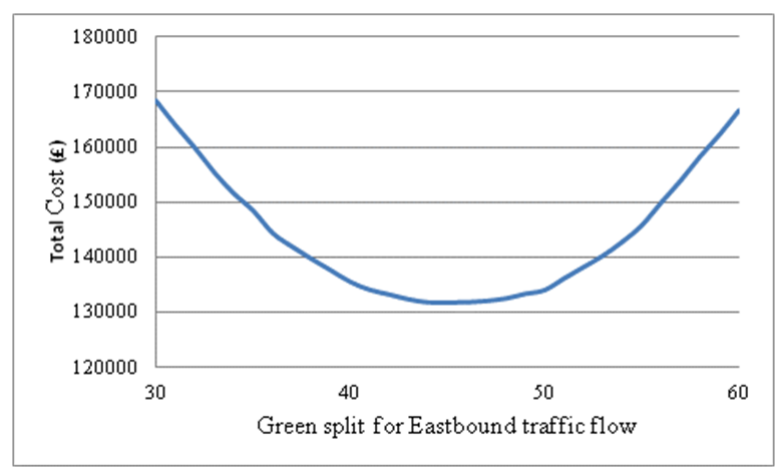

Figure 6. Total cost for different green splits

\subsection{Examples of evaluating maintenance using optimisation}

After the method has been applied to show its potential in maintenance planning, more complex situations of maintenance decisions were considered. Further research has been carried out in applying optimisation techniques, such as genetic algorithms, in order to search for the best maintenance strategy for the network. Two conflicting parties were considered - road users and highway agency, since performing road maintenance at night can be convenient for road users but costly for highways agency. If the additional road user costs due to maintenance were acceptable, daytime maintenance shift would be more preferable to highways agencies, as the safety of road workers and the quality of maintenance work are both enhanced. As a consequence, maintenance arrangements, i.e. maintenance start time, speed restrictions, green split times, etc., were optimised, so that and a balance between road user costs and maintenance costs could be achieved. For example, some results showed that pausing maintenance works during the peak hours can reduce the road user cost, and therefore, give a better balance between the maintenance cost, since no works are carried out at night, and the road user cost, since traffic delays on the network are reduced. This example illustrates the suitability of the proposed model to inform maintenance planning decisions, when an optimisation technique is employed to search for best solutions.

\section{CONCLUSIONS AND FUTURE WORK}

A macroscopic road network flow model has been proposed in this paper. It is used to calculate the traffic flow through the network and the queues which occur and disperse throughout the day. This model is an advancement of the previously developed models of this type due to the following features:
- Both urban and motorway roads are considered in the same model, which can represent interactions between the two types of roads.

- Two-way traffic is modelled using an iterative simulation method to take account of dependent flows in the network.

- Traffic exiting or joining an urban link, such as housing estates and work place locations, is modelled as sink or source node respectively.

- A shared lane is used to model traffic interaction among mixed directional flow.

- Roadworks on the network are modelled, which are used to compare different maintenance arrangements and their effects on the network.

The results presented illustrate the capability of the model to describe the evolution of dependent traffic flows and queue dynamics throughout the network, as close to reality as reasonably practicable. Also, it is shown that the model can be used to analyse maintenance effects on the network, to evaluate different maintenance arrangements in terms of network congestion and to support maintenance planning decisions.

In order to address potential processing time issues, when larger networks are analysed, the performance of the algorithm could be improved by minimising the time spent to evaluate the network. Also, the model could be used for further applications in road maintenance planning. For example, in addition to maintenance cost and road user cost, road condition data could be used in the analysis, and different types of maintenance for a specific condition could be proposed, while maintenance effects are evaluated using the proposed model.

\section{ACKNOWLEDGMENT}

John Andrews is The Royal Academy of Engineering and Network Rail Professor of Infrastructure Asset Management. He is also Director of The Lloyd's Register Foundation* Centre for Risk and Reliability Engineering at the University of Nottingham. Rasa Remenyte-Prescott is The Lloyd's Register Foundation Lecturer in Risk and Reliability Engineering at the University of Nottingham. Both authors gratefully acknowledge the support of the appropriate organisations.

* The Lloyd's Register Foundation (LRF) supports the advancement of engineering-related education, and funds research and development that enhances safety of life at sea, on land and in the air.

\section{REFERENCES}

A.I.A. 2011. Annual Local Authority Road Maintenance Survey. HMPR Limietd: London 
Breton, P., Hegyi, A., De Schutter, B. \& Helendoorn, H. 2002. Shock Wave Elimination/Reduction by Optimal Coordination of Variable Speed Limits. Intelligent Transportation Systems, 2002. Proceedings. The IEEE 5th International Conference on: 225-230.

Daganzo, C.F. 1994. The Cell Transmission Model - a Dynamic Representation of Highway Traffic Consistent with the Hydrodynamic Theory, Transportation Research Part B Methodological 28(4): 269-287.

Deflorio, F. P. 2003. Evaluation of a Reactive Dynamic Route Guidance Strategy, Transportation Research Part C: Emerging Technologies 11(5): 375-388.

DfT, 2012. WebTAG: Unit 3.5.6 Values of Time and Operating Costs, Department of Transport

Highways Agency, 2011. Traffic Information Database, Highways Agency

Hegyi, A., De Shutter, B. \& Hellendoorn, J. 2005. Optimal Coordination of Variable Speed Limits to Suppress Shock Waves, Intelligent Transportation Systems, IEEE Transactions on 6(1): 102-112.

Karimi, A., Hegyi, A., De Schutter, B., Hellendoorn, H. \& Middelham, F. 2004. Integration of Dynamic Route Guidance and Freeway Ramp Metering using Model Predictive Control, American Control Conference, 2004. Proceedings of the 2004 6: 5533-5538.

Lighthill, M.J. \& Whitham, G.B. 1955. On Kinematic Waves. II. A Theory of Traffic Flow on Long Crowded Roads. Proceedings of the Royal Society of London. Series A. Mathematical and Physical Sciences, 229(1178): 317-345.

Messmer, A. \& Papageorgiou, M. 1990. METANET: A Macroscopic Simulation Program for Motorway Networks, Traffic Engineering and Control 31(9): 466-470.

Van den Berg, M., Hegyi, A., De Schutter, B. \& Hellendoorn, J. 2007. Integrated Traffic Control for Mixed Urban and Freeway Networks: A Model Predictive Control Approach, European Journal of Transportation and Infrastructure Research 3(3): 223-250. 